MARTINS, G. L. M.; TOMQUELSKI, G. V.; PAPA, G. Aplicação de acybenzolar-s-methyl em algodoeiro para controle de Aphis gossypii (Glover) e Ramularia areola (Atkinson). Revista de Agricultura Neotropical, Cassilândia-MS, v. 2, n. 1, p. 53-59, jan./mar. 2015.

\title{
APLICAÇÃO DE ACYBENZOLAR-S-METHYL EM ALGODOEIRO PARA CONTROLE DE Aphis gossypii (GLOVER) E Ramularia areola (ATKINSON)
}

\section{GUSTAVO LUÍS MAMORÉ MARTINS ${ }^{1}$, GERMISON VITAL TOMQUELSKI ${ }^{2}$, GERALDO PAPA ${ }^{3}$}

\footnotetext{
${ }^{1}$ Universidade Estadual de Mato Grosso do Sul, Cassilândia/MS, Brasil, gustavomamore@yahoo.com.br, ${ }^{2}$ Fundação Chapadão, Chapadão do Sul/MS, Brasil, germisont@fundacaochapadao.com.br, ${ }^{3}$ Universidade Estadual Paulista "Júlio de Mesquita Filho", Ilha Solteira/SP, Brasil, gpapa@feis.unesp.br
}

RESUMO: O acibenzolar-S-methyl funciona como um ativador na resistência das plantas contra pragas e doenças. O objetivo do trabalho foi avaliar a atividade de acybenzolar-Smethyl sobre Aphis gossypii (Glover) e Ramularia areola (Atkinson) em algodoeiro. O experimento foi conduzido no município de Selvíria, MS. O delineamento experimental foi de blocos casualizados no esquema de parcelas subdivididas $(2 \times 6)$, constituídos pela combinação de duas cultivares de algodão (Acala-90 e DeltaOpal) e seis indutores de resistência. Os tratamentos foram: 1) testemunha; 2) acybenzolar-s-methyl (20 g i.a. ha $\left.\left.{ }^{-1}\right) ; 3\right)$ acybenzolar-smethyl + azoxystrobin $\left(20+40\right.$ g i.a. ha $\left.\left.{ }^{-1}\right) ; 4\right)$ acybenzolar-s-methyl + tiofanato metílico $(20+$ $350 \mathrm{~g}$ i.a. ha $\left.\left.{ }^{-1}\right) ; 5\right)$ azoxystrobin $\left(40 \mathrm{~g}\right.$ i.a. $\left.\mathrm{ha}^{-1}\right)$ e 6$)$ tiofanato metílico $\left(350 \mathrm{~g}\right.$ i.a. ha $\left.{ }^{-1}\right)$. A avaliação de $A$. gossypii foi realizada com intervalo de 10 dias após a aplicação do indutor. Foi analisada a severidade de $R$. areola aos 101, 109 e 116 dias após a emergência, em 10 plantas marcadas na parcela. Os resultados mostram que a aplicação de acybenzolar-s-methyl, isolada ou em conjunto com fungicidas, na dose de $20 \mathrm{~g}$ i.a. ha ${ }^{-1}$, reduziu a população de $A$. gossypii. O acybenzolar-s-methyl ( $20 \mathrm{~g}$ i.a. $\left.\mathrm{ha}^{-1}\right)$ proporcionou menor incidência de $R$. areola.

PALAVRAS-CHAVE: Gossypium hirsutum, pulgão, ramulária, indutor de resistência.

\section{APLICATION OF ACYBENZOLAR-S-METHYL ON COTTON CROP TO CONTROL OF Aphis gossypii (GLOVER) AND Ramularia areola (ATKINSON)}

\begin{abstract}
Acibenzolar-S-methyl acts as an activator of natural plant defenses, inducing plant resistance against pests and diseases. The objective of this study was to evaluate the activity of acybenzolar-S-methyl on Aphis gossypii (Glover) and Ramularia areola (Atkinson) in the cotton crop. The experiment was conducted in Selvíria (MS). The experimental design was randomized blocks with plots in a factorial $(2 \times 6)$, constituted by the combination of two cotton cultivars (Acala-90 and DeltaOpal) and six treatments of resistance. The treatments were: 1) control, 2) acybenzolar-s-methyl (20 g i.a. ha $\left.{ }^{-1}\right), 3$ ) acybenzolar-S-methyl + azoxystrobin $\left(20+40 \mathrm{~g}\right.$ i.a ha $\left.{ }^{-1}\right)$, 4) acybenzolar-s-methyl + thiophanate methyl $\left(20+350 \mathrm{~g}\right.$ i.a. ha $\left.\left.{ }^{-1}\right), 5\right)$ azoxystrobin $\left(40 \mathrm{~g}\right.$ i.a. ha $\left.{ }^{-1}\right)$ and 6) thiophanate methyl (350 g i.a. ha ${ }^{-1}$ ). Aphis gossypii was evaluated with an interval of 10 days after the application of treatments. The severity of $R$. areola at 101, 109 and 116 days after emergence was analyzed in ten plants marked in the plot. The results show that applying acybenzolar-smethyl, alone or in combination with fungicides, the rates of $20 \mathrm{~g}$ i.a. ha ${ }^{-1}$ reduced the population of A. gossypii. The acybenzolar-S-methyl $\left(20 \mathrm{~g}\right.$ i.a. $\left.\mathrm{ha}^{-1}\right)$ provided the lowest incidence of $R$. areola.
\end{abstract}

KEY WORDS: Gossypium hirsutum, aphid, ramularia, resistance inducer. 


\section{INTRODUÇÃO}

Em algodoeiro é relatado grande número de pragas (TOMQUELSKI; MARTINS, 2013) e doenças (CIA; SALGADO, 1997) que durante o ciclo da cultura são capazes de causarem redução na produção, resultando em prejuízos para o cotonicultor. Entre os insetospragas, cita-se o pulgão-do-algodoeiro, Aphis gossypii (GLOVER, 1877) que é uma das primeiras pragas que aparece na cultura.

Esses insetos vivem nas folhas ou brotos novos, sugando a seiva das plantas, causando encarquilhamento das folhas e deformação dos brotos. A praga ao se alimentar das plantas, transmitem vírus que causam o vermelhão do algodoeiro e o mosaico das nervuras forma Ribeirão Bonito (TOMQUELSKI, 2014). A ramulária, doença causada pelo fungo Ramularia areola (Atkinson), ocasionam as folhas manchas angulosas que variam de 1 a $4 \mathrm{~mm}$, circunscrita pelas nervuras de coloração branca ou amarelada e aspecto farináceo/pulverulento (CIA; SALGADO, 1997).

Uma das respostas das plantas contra o ataque de moléstias está relacionada à resistência sistêmica adquirida (SAR - Systemic Acquired Resistance). Foi introduzido o indutor acibenzolar-S-methyl no mercado brasileiro que funciona como um ativador das defesas naturais das plantas (TOMQUELSKI et al., 2007). O éster 2-metil benzo-(1,2,3)tiadiazole-7-carbotióico, de nome químico acibenzolar-S-methyl (ASM) é o composto mais estudado e mais eficiente que pode levar à ativação de genes que codificam a resistência de plantas contra pragas e doenças (KESSMANN et al., 1994). Além de não apresentar fitotoxidez em plantas, o composto é facilmente translocado pelos tecidos da planta de forma sistêmica (OOSTENDORP et al., 2001; COSTA; MORAES, 2006).

A utilização de compostos bioativos com capacidade de proteger as plantas contra doenças possibilitando a expressão de mecanismos de defesa latentes é uma estratégia que vem sendo amplamente pesquisada (BRISSET et al., 2000; COQUEIRO et al., 2011) no controle de pragas e doenças em culturas agrícolas (SAWANT et al., 1994; CARVALHO et al., 1999; ANDRADE E ANDRADE, 2001).

Em vista do potencial de acybenzolar-s-methyl no manejo de pragas e doenças em algodoeiro, o objetivo da pesquisa foi avaliar o efeito desse indutor de resistência sobre Aphis gossypii e Ramularia areola em algodoeiro.

\section{MATERIAL E MÉTODOS}

O experimento foi conduzido em Latossolo Vermelho distrófico (LVd), localizado na fazenda experimental da Faculdade de Engenharia de Ilha Solteira - Unesp, em Selvíria, Estado do Mato Grosso do Sul $\left(20^{\circ} 22^{\prime}\right.$ S e $\left.51^{\circ} 22^{\prime} \mathrm{W}\right)$, com altitude média de $335 \mathrm{~m}, 1.370$ $\mathrm{mm}$ de precipitação média anual, $23,5^{\circ} \mathrm{C}$ de temperatura média anual, umidade relativa variando entre $70 \%$ e $80 \%$, como média anual. O ensaio foi conduzido no período de janeiro a julho de 2004.

O delineamento experimental foi de blocos casualizados em esquema de parcelas subdivididas (2×6), constituídos pela combinação de duas cultivares de algodão (Acala-90 e DeltaOpal) e seis indutores de resistência, com suas respectivas doses e épocas de aplicação (Tabela 1).

As parcelas experimentais foram constituídas por 8 linhas da cultura com $7 \mathrm{~m}$ de comprimento, sendo as 6 linhas centrais consideradas como área útil para avaliações. 
Tabela 1. Produtos utilizados, com suas respectivas doses e época de aplicação em dias após a emergência (DAE). Selvíria, MS, 2004.

\begin{tabular}{lcc}
\hline Tratamento & $\begin{array}{c}\text { Dose } \\
\left(\mathrm{g} \mathrm{i} . \mathrm{a} \cdot \mathrm{ha}^{-1}\right)\end{array}$ & $\begin{array}{c}\text { Época de aplicação } \\
(\mathrm{DAE})\end{array}$ \\
\hline 1- Testemunha & - & - \\
2- Acybenzolar-S-methyl & 20 & $40,50,60$ \\
3- Acybenzolar-S-methyl + Azoxystrobin & $20+40$ & $40,50,60+40,94^{*}$ \\
4- Acybenzolar-S-methyl + Tiofanato metilico & $20+350$ & $40,50,60+40,94^{*}$ \\
5- Azoxystrobin & 40 & $40,94^{*}$ \\
6- Tiofanato metilico & 350 & $40,94^{*}$ \\
\hline
\end{tabular}

*Constatação de sintomas iniciais de ramularia no terço inferior das plantas.

$\mathrm{Na}$ adubação de semeadura foram aplicados $20 \mathrm{~kg} \cdot \mathrm{ha}^{-1}$ de N, $92 \mathrm{~kg} \mathrm{ha}^{-1}$ de $\mathrm{P}_{2} \mathrm{O}_{5}$ e 40 $\mathrm{kg} \mathrm{ha}^{-1}$ de $\mathrm{K}_{2} \mathrm{O}$, e cobertura $70 \mathrm{~kg} \mathrm{ha}^{-1}$ de $\mathrm{N}$ e $80 \mathrm{~kg} \mathrm{ha}^{-1}$ de $\mathrm{K}_{2} \mathrm{O}$. A semeadura foi realizado em 23/12/2003, com espaçamento de 0,85 entre linhas, e sementes necessárias para a obtenção de 8-9 plantas $\mathrm{m}^{-1}$, sendo que antes da semeadura, as sementes foram tratadas com thiametoxam e carbendazim + thiram nas doses de 240, $90+210$ g i.a. por $100 \mathrm{~kg}$ de sementes, respectivamente. A infestação das pragas e patógenos ocorreram naturalmente.

Aos 81 dias após a emergência realizou-se uma aplicação de thiametoxan na dose de $25 \mathrm{~g}$ i.a. ha ${ }^{-1}$ para o controle de pulgões (A. gossypii). O controle de outras pragas foi realizado com pulverizações de deltametrina, na dose de $10 \mathrm{~g}$ i.a. ha ${ }^{-1}$ e betacyflutrina, na dose de 12,5 g i.a. ha ${ }^{-1}$, em intervalos de 7 dias, iniciando aos 50 e até os 120 dias após a emergência. $\mathrm{O}$ controle de ácaros na área foi realizado com 2 pulverizações de dicofol na dose de $720 \mathrm{~g}$ i.a. ha ${ }^{-1}$, sendo a primeira aos 65 e a segunda aos 80 dias após a emergência.

As avaliações de pulgão (A. gossypii) foram realizadas previamente as aplicações, com intervalo de 10 dias após a aplicação do indutor, sendo que na última aplicação do indutor foi realizada avaliação aos 10 e 20 dias após a aplicação.

Foi analisada a severidade da mancha de ramulária ( $R$. areola), aos 101, 109 e 116 dias após a emergência, em 10 plantas marcadas previamente sem sintoma, por parcela, sendo que, para análise foi usada a escala de notas proposta por Iamamoto (2003), sendo: 1 - sem sintomas; 2 - até $5 \%$ de sintomas na planta; 3 - até $15 \%$ de sintomas na planta com queda de folhas axilares no terço inferior; 4 - até $30 \%$ de sintomas na planta, com queda de folhas axilares no terço médio, início de desfolha precoce no baixeiro e lesões de ramulária no ponteiro; 5 - acima de $50 \%$ de sintomas na planta, com queda de folhas no terço superior e desfolha precoce.

Os dados obtidos foram submetidos à análise de variância pelo teste $\mathrm{F}$ e as médias comparadas pelo teste de Tukey (5\%) com uso do Programa Sisvar versão 5.1 Build 72 (FERREIRA, 2011).

\section{RESULTADOS E DISCUSSÃO}

Quanto ao número de A. gossypii, aos 50 dias após a emergência o tratamento com acybenzolar aplicado isoladamente na dose de $20 \mathrm{~g}^{\text {i.a. }}$ ha $^{-1}$ apresentou o menor número de pulgões. Aos 60 e 70 dias após a emergência, as menores populações de pulgões foram constatadas nos tratamentos com acybenzolar + azoxystrobim nas doses de 20 g i.a. ha ${ }^{-1}+40$ g i.a. ha ${ }^{-1}$ e acybenzolar + tiofanato metilico na dose de 20 g i.a. ha ${ }^{-1}+350$ g i.a. ha ${ }^{-1}$ (Tabela 2). Esses resultados são semelhantes aos encontrados por Correa et al. (2004), que observaram 
que a aplicação de acybenzolar reduziu a população de mosca branca, Bemisia tabaci biótipo B, na cultura do pepino pela redução da oviposição, aumento de ciclo e mortalidade na fase de ninfas, apresentando-se como alternativa para o manejo integrado de mosca branca.

Tabela 2. Valores do teste $\mathrm{F}$ obtidos na análise de variância e número de A. gossypii por tratamento em diferentes épocas, em função de diferentes variedades e diferentes usos do indutor de resistência. Selvíria, MS. 2004.

\begin{tabular}{|c|c|c|c|c|c|}
\hline Tratamentos & $\begin{array}{c}40 \\
\text { Prévia }\end{array}$ & 50 & 60 & 70 & 80 \\
\hline 1-testemunha & 34 & $243 \mathrm{ah}$ & 4166 a & 41609 & 10745 \\
\hline 2- acybenzolar & $4,6 \mathrm{a}$ & $11,6 \mathrm{~b}$ & $246,3 \mathrm{ab}$ & $245,0 \mathrm{ab}$ & $1804,4 \mathrm{a}$ \\
\hline 3- acybenzolar + azoxystrobim & $7,8 \mathrm{a}$ & $13,5 \mathrm{ab}$ & $210,4 b$ & $210,6 \mathrm{ab}$ & $1878,8 \mathrm{a}$ \\
\hline 4- acybenzolar + tiofanato metilico & $6,1 \mathrm{a}$ & $18,3 \mathrm{ab}$ & $206,5 \mathrm{~b}$ & $201,3 \mathrm{~b}$ & $1860,0 \mathrm{a}$ \\
\hline 5- azoxystrobim & $4,0 \mathrm{a}$ & $30,8 \mathrm{a}$ & $265,0 \mathrm{ab}$ & $288,8 \mathrm{ab}$ & $1872,5 \mathrm{a}$ \\
\hline 6- tiofanato metilico & $8,9 \mathrm{a}$ & $26,1 \mathrm{ab}$ & $369,4 \mathrm{ab}$ & $373,8 \mathrm{ab}$ & $1952,5 \mathrm{a}$ \\
\hline Delta opal & $5,6 \mathrm{a}$ & $19,8 \mathrm{a}$ & 277,6 a & $301,0 \mathrm{a}$ & $1947,5 \mathrm{a}$ \\
\hline Acala-90 & $6,0 \mathrm{a}$ & $21,7 \mathrm{a}$ & 293,8 a & $277,7 \mathrm{a}$ & $1832,7 \mathrm{~b}$ \\
\hline $\begin{array}{l}\text { Valores de F } \\
\text { variedades }\end{array}$ & $0,05^{\mathrm{ns}}$ & $0,30^{\mathrm{ns}}$ & $0,19^{\mathrm{ns}}$ & $0,37^{\mathrm{ns}}$ & $4,59^{*}$ \\
\hline $\mathrm{CV}(\%)$ & 114,86 & 56,27 & 45,03 & 45,79 & 9,82 \\
\hline Média geral & 5,8 & 20,8 & 285,8 & 289,4 & 1890,1 \\
\hline
\end{tabular}

Houve interação significativa entre tratamento e variedades apenas nas avaliações de severidade de $R$. areola aos 109 e 116 dias após a emergência (Tabela 3). Durante o período do experimento a precipitação não foi regular, com intervalos superiores a 20 dias sem chuva, o que não favoreceu o desenvolvimento do fungo $R$. areola na fase inicial do florescimento, que segundo Hillocks (1992) a ocorrência de umidade relativa elevada (acima de 85\%), alta pluviosidade, noites úmidas seguidas dias com temperatura de 25 a $30{ }^{\circ} \mathrm{C}$ são condições favoráveis ao fungo.

Tabela 3. Valores do teste F obtidos na análise de variância e médias das notas por parcela da severidade de Ramularia areola, em diferentes épocas, em função de diferentes variedades e diferentes usos do indutor de resistência. Selvíria, MS. 2004.

\begin{tabular}{|c|c|c|c|}
\hline Tratamentos & 101 dae & 109 dae & 116 dae \\
\hline & & --médias- & ------------- \\
\hline 1- testemunha & $1,28 \mathrm{a}$ & - & - \\
\hline 2- acybenzolar & $1,06 \mathrm{ab}$ & - & - \\
\hline 3- acybenzolar + azoxystrobim & $1,00 \mathrm{~b}$ & - & - \\
\hline 4- acybenzolar + tiofanato metilico & $1,03 \mathrm{~b}$ & - & - \\
\hline 5- azoxystrobim & $1,03 \mathrm{~b}$ & - & - \\
\hline 6- tiofanato metílico & $1,15 \mathrm{ab}$ & - & - \\
\hline Delta opal & $1,03 \mathrm{~b}$ & - & - \\
\hline Acala-90 & $1,15 \mathrm{a}$ & - & - \\
\hline $\begin{array}{l}\text { Valores de F } \\
\text { variedades }\end{array}$ & $8,67^{* *}$ & $39,17^{* *}$ & $47,33^{* *}$ \\
\hline $\mathrm{CV}(\%)$ & 13,05 & 11,91 & 14,93 \\
\hline Média geral & 1,09 & 1,18 & 1,37 \\
\hline
\end{tabular}


O desdobramento da interação entre tratamentos e cultivares (Tabela 4), revelou que os tratamentos com acybenzolar + azoxystrobim e acybenzolar + tiofanato metilico, quando avaliado em 10 plantas marcadas por parcela, apresentaram menor severidade de $R$. areola em ambas as cultivares utilizadas.

Constatou-se ainda que a severidade de $R$. areola apresentou comportamento diferenciado quanto a cultivar utilizada sendo que, os maiores valores foram encontrados na cultivar Acala-90 (Tabelas 3). Os resultados concordam com Iamamoto (2003), que cita as cultivares usadas no experimento, como suscetíveis a doença, entretanto a cultivar DeltaOpal apresenta resistência.

Tabela 4. Efeito de diferentes tratamentos em duas variedades de algodão, na severidade da mancha de ramulária (Ramularia areola). Médias de notas por tratamento. Selvíria, MS, 2004.

\begin{tabular}{|c|c|c|c|c|c|}
\hline \multirow[t]{2}{*}{ Tratamentos } & \multirow{2}{*}{$\begin{array}{l}\text { Dose em } \\
\text { g.i.a..ha }{ }^{-1}\end{array}$} & \multicolumn{2}{|c|}{109 d.a.e. } & \multicolumn{2}{|c|}{116 d.a.e. } \\
\hline & & DeltaOpal & Acala-90 & DeltaOpal & Acala-90 \\
\hline 1-testemunha & 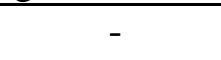 & $1,2 \mathrm{Ba}$ & $1,8 \mathrm{Aa}$ & $1,5 \mathrm{Ba}$ & $2,1 \mathrm{Aa}$ \\
\hline 2- acybenzolar & 20 & $1,1 \mathrm{Ba}$ & $1,3 \mathrm{Abc}$ & $1,1 \mathrm{Bab}$ & 1,6 Abc \\
\hline $\begin{array}{l}\text { 3- acybenzolar + } \\
\text { azoxystrobim }\end{array}$ & $20+40$ & $1,0 \mathrm{Aa}$ & $1,1 \mathrm{Ac}$ & $1,0 \mathrm{Ab}$ & $1,1 \mathrm{Ad}$ \\
\hline $\begin{array}{l}\text { 4- acybenzolar + } \\
\text { tiofanato metilico }\end{array}$ & $20+350$ & $1,0 \mathrm{Aa}$ & $1,1 \mathrm{Ac}$ & $1,0 \mathrm{Ab}$ & 1,1 Acd \\
\hline 5- azoxystrobim & 40 & $1,0 \mathrm{Aa}$ & $1,2 \mathrm{Abc}$ & $1,0 \mathrm{Ab}$ & 1,3 Acd \\
\hline 6- tiofanato metilico & 350 & $1,0 \mathrm{Ba}$ & 1,5 Aab & $1,1 \mathrm{Bab}$ & 1,9 Aab \\
\hline
\end{tabular}

De maneira semelhante, Andrade \& Andrade (2001) ao avaliar os efeitos do acybenzolar como indutor de resistência em plantas de algodoeiro, na cultivar Acala-90, sobre o fungo $R$. areola,observaram que o tratamento com acybenzolar 20 g.i.a..ha ${ }^{-1}$ e mais uma aplicação de difenoconazole 75 g.i.a..ha ${ }^{-1}$, realizada no aparecimento da doença no terço médio ( $5 \%$ de área foliar lesionada no terço médio) apresentou menor severidade da doença e maior produção de algodão em caroço.

Em outra pesquisa realizada com a cultura do tomateiro, Graves (2001) concluiu que a utilização de acybenzolar foi tão efetiva, quanto o uso de oxicloreto de cobre no controle de Xanthomonas campestris, relatando também ser uma alternativa viável, para a diminuição da contaminação do ambiente.

Desse modo, os resultados obtidos nessa pesquisa sugerem que a utilização de acybenzolar-s-methyl é uma opção potencial no manejo do pulgão e ramulária em algodoeiro.

\section{CONCLUSÕES}

A aplicação de acybenzolar-s-methyl, isolada ou em conjunto com fungicidas, na dose de 20 g i.a ha ${ }^{-1}$, reduziu a população de $A$. gossypii.

O acybenzolar-s-methyl na dose de 20 g.i.a ha ${ }^{-1}$ proporcionou menor severidade de $R$. areola.

\section{REFERÊNCIAS BIBLIOGRÁFICAS}

ANDRADE, D. F. A. A.; ANDRADE, P. J. M. Avaliação da eficiência do ativador de defesa vegetal "Bion 500 WG" como indutor de resistência à mancha foliar de ramulária 
(Ramularia areola) no algodoeiro. Dourados-MS: Embrapa, 2001. p. 99-102. (Documentos, 20).

BENELLI, A. I. H.; DENARDIN, N. D.; FORCELINI, C. A. Ação do acibenzolar-S-metil aplicado em tubérculos e plantas de batata contra canela preta, incitada por Pectobacterium carotovorum subsp. atrosepticum atípica. Fitopatologia Brasileira, Brasília-DF, v. 29, n. 3, p. 263-267, 2004.

BRISSET, M. N.; CESBRON, S.; THOMSON, S. V.; PAULIN, J. P. Acibenzolar-S-methyl induces the accumulation of defense-related enzymes in apple and protects from fire blight. European Journal of Plant Pathology, Netherlands, v. 106, n. 6, p. 529-536, 2000.

CARVALHO, S. P.; MORAES, J. C.; CARVALHO, J. G. Efeito do silício na resistência do sorgo (Sorghum bicolor) ao pulgão-verde Schizaphis graminum (Rond.) (Homoptera: Aphididae). Anais da Sociedade Entomológica do Brasil, Londrina-PR, v. 28, n. 1, p. 505510, 1999.

CIA, E.; SAlGADO, C. L. Doenças do algodoeiro. In: KIMATI, H.; AMORIM, L.; BERGAMIN FILHO, A.; CAMARGO, L. E. A.; REZENDE, J. A. M. (Eds.) Manual de Fitopatologia: doenças das plantas cultivadas. 3. ed. Piracicaba-SP: Ceres, 1997. v. 2. p. 334.

COQUEIRO, D. S. O.; SILVA, C. N.; CERQUEIRA-SILVA; CARLOS-BERNARD, M.; LIMA, G. S. A.; SANTOS, A.; OLIVEIRA, A. C. Aplicação de suspensões de Agaricus blazei, Lentinula edodes e de acibenzolar-S-metil na redução da antracnose em frutos de maracujá-azedo. Tropical Plant Pathology, Brasília-DF, v. 36, n. 1, p. 54-59, 2011.

COSTA, R. R.; MORAES, J. C. Efeitos do ácido silícico e do acibenzolar-S-methyl sobre Schizaphis graminum (Rondani) (Hemiptera: Aphididae) em plantas de trigo. Neotropical Entomology, Londrina-PR, v. 35, n. 6, p. 834-839, 2006.

FERREIRA, D. F. Sisvar: A computer statistical analysis system. Ciência e Agrotecnologia, Lavras-MG, v. 35, n. 6, p. 1039-1042, 2011.

GRAVES, A. S. Reducing copper and chlorotalonil in staked tomato production on Virginia's Eastern Shore. 2001. 89 p. Dissertação (Master em Fitopatoliga) - State University of Virginia, Blacksburg, 2001.

HILLOCKS, R. J. Fusarium wilt. In: HILLOCKS, R. J. (Ed.). Cotton diseases. Wallington: CAB International, 1992. p. 127-160.

KESSMANN, H.; STAUB, T.; HOFFMANN, C.; MAETZKE, T.; HERZOG, J.; WARD, E.; UKNES, S.; RYALS. J. Induction of systemic acquired disease resistance in plants by chemicals. Annual Review of Phytopathology, Palo Alto, v. 32, p. 439-459, 1994.

OOSTENDORP, M.; KUNZ, W.; DIETRICH, B.; STAUB, T. Induced disease resistance in plants by chemicals. European Journal of Plant Pathology, Netherlands, v. 107, p. 19-28, 2001.

SAWANT, A. S.; PATIL, V. H.; SAVANT, N. K. Rice hull ash applied to seedbed reduces deadhearts in transplanted rice. International Rice Research, Manila, v. 19, n. 4, p. 21-22. 1994.

TOMQUELSKI, G. V. Aspecto e manejo de pragas do algodoeiro. Pesquisa, Tecnologia e Produtividade, Chapadão do Sul-MS, v. 1, n. 2, p. 31-29, 2014. 
TOMQUELSKI, G. V.; MARTINS, G. L. M.; PAPA, G. Efeito dos indutores de resistência acibenzolar-s-metil e silício na biologia de Alabama argillacea (Lepidotera: Noctuidae) em algodoeiro. Revista de Agricultura, Piracicaba-SP, v. 82, p. 170-175, 2007.

TOMQUELSKI, G. V.; MARTINS, G. L. M. Pragas em algodoeiro na região dos Chapadões. Safra 2012/2013. Pesquisa, Tecnologia e Produtividade, Chapadão do Sul-MS, v. 1, n. 1, p. 25-51, 2013. 